\title{
The long non-coding RNA CYTOR drives colorectal cancer progression by interacting with NCL and Sam68
}

Xue Wang ${ }^{1,2}$, Hongfei Yu ${ }^{1}$, Wenjie Sun ${ }^{1}$, Jianlu Kong ${ }^{1}$, Lei Zhang ${ }^{2}$, Jinlong Tang ${ }^{1}$, Jingyu Wang ${ }^{1}$, Enping Xu ${ }^{1}$, Maode Lai ${ }^{1,2^{*}}$ and Honghe Zhang ${ }^{1 *}$

\begin{abstract}
Background: Long non-coding RNAs (IncRNAs) function as key molecules in cancer progression. The IncRNA CYTOR plays oncogenic roles in multiple types of cancer, yet the detailed molecular mechanisms of those roles remain unknown. The aim of this study was to investigate the clinical significance, biological function and interacting partners of CYTOR in colorectal cancer (CRC).

Methods: A systematic and comprehensive analysis of CYTOR expression was performed in 138 CRC samples and in the TCGA and GEO databases. Biological function was investigated through knockdown and overexpression of CYTOR in vitro and in vivo. In addition, its protein binding partner was identified and validated using ChIRP-MS and RNA immunoprecipitation assays. Their key interaction sites on CYTOR were verified by CRISPR/Cas9 and a series of mutant constructs. Furthermore, the downstream targets of CYTOR were confirmed via immunoblotting and luciferase reporter assays.

Results: CYTOR was significantly up-regulated in CRC samples and associated with poor prognosis, promoting proliferation and metastasis in vitro and in vivo. NCL and Sam68 could recognize their specific motifs and directly bind to EXON1 of CYTOR. Moreover, EXON1 was the key functional site mediating the interaction of CYTOR with $\mathrm{NCL}$ and Sam68. NCL and Sam68 functioned as oncogenes to promote CRC progression. Furthermore, we confirmed that the heterotrimeric complex of CYTOR, NCL and Sam68 activated the NF-KB pathway and EMT to contribute to CRC progression.
\end{abstract}

Conclusion: CYTOR plays important roles in CRC progression by interacting with NCL and Sam68 and may serve as a prognostic biomarker and/or an effective target for CRC therapies.

Keywords: Colorectal cancer, CYTOR, NCL, Sam68

\section{Background}

It is now widely accepted that cancer progression results from the gradual accumulation of genetic mutations and epigenetic alterations that successively increase cell proliferation, motility and stemness $[1,2]$. Long non-coding RNAs (lncRNAs), since their recent discovery, have gained widespread attention as a new player of epigenetic regulation in various cellular and biological processes including gene regulation and chromatin dynamics; and the aberrant

\footnotetext{
*Correspondence: Imp@zju.edu.cn; honghezhang@zju.edu.cn

${ }^{1}$ Department of Pathology, Key Laboratory of Disease Proteomics of Zhejiang Province, Zhejiang University School of Medicine, Hangzhou 310058, China Full list of author information is available at the end of the article
}

expression and mutations of lncRNAs are closely linked to tumorigenesis, metastasis, and tumor stage [3].

Colorectal cancer (CRC) is a frequently diagnosed malignancy and the 3rd leading cause of cancer-related mortality in the western world. The progression of CRC is fast, and untreated tumors rapidly disseminate and form metastatic foci. Therefore, further understanding the mechanisms that drive the disease and identifying diagnostic and therapeutic targets are priorities for improving CRC patients' outcome [4]. To date, several important lncRNAs such as UPAT [5], CCAT1 [6], CCAL [7], LINC01133 [8], LET [9] and HOTAIR [10] have been shown to contribute to CRC development

(C) The Author(s). 2018 Open Access This article is distributed under the terms of the Creative Commons Attribution 4.0 International License (http://creativecommons.org/licenses/by/4.0/), which permits unrestricted use, distribution, and 
and could be used as new candidates for diagnostics and therapy. Previously, we found through laser microdissection capture in CRC tissues that a novel lncRNA, CYTOR (also known as LINC00152), was up-regulated in tumor budding cells [11]. Recently, the aberrant expression of CYTOR has been reported in some types of cancers including gastric cancer [12], hepatocellular carcinoma [13], colon cancer [14], gallbladder cancer [15] and renal cell carcinoma [16], in which it may act as an oncogene. However, the detailed mechanism of action of CYTOR in CRC progression remains largely unknown.

NCL (nucleolin) and Sam68 (KHDRBS1) belong to the category of RNA-binding proteins (RBPs), which control RNA metabolism and biogenesis-including RNA synthesis, pre-RNA splicing, RNA processing, ribosomal assembly and maturation-and play multiple roles in cancer development $[17,18]$. Nevertheless, it is unclear whether NCL and Sam68 are involved in lncRNA, in particular, whether they function along with CYTOR to regulate CRC progression. In this study, we performed a comprehensive survey of CYTOR expression in CRC samples from online databases and our tissue bank and clarified the roles that CYTOR plays in CRC progression by interacting with NCL and Sam68.

\section{Methods}

\section{Clinical materials}

Two cohorts of clinical samples from the tissue bank in our laboratory were used in this study according to protocols approved by the Internal Review Board of Zhejiang University. A total of 138 pairs of CRC tissues and matched normal tissues were collected to measure CYTOR levels by qRT-PCR. Each experiment was performed at least three times. Another cohort, including 144 CRC FFPE tissue samples, was used for the detection of NCL and Sam68 expression by immunohistochemistry (IHC), and the IHC scores were evaluated by at least two pathologists. The pathological diagnoses of all samples were evaluated by pathologists. All patients with familial adenomatous polyposis, hereditary non-polyposis CRC, or inflammatory bowel disease were excluded. All tissue samples were obtained from colorectal adenocarcinoma patients without any radiotherapy, chemotherapy, or other adjuvant treatment prior to surgery and diagnosis.

\section{Public databases}

The transcriptome expression profiling of CRC and relevant clinical information in this study were identified by searching public databases online. The key words "colon cancer or colorectal cancer and expression profiling by array" were used to search the GEO databases. In addition, the data from the TCGA database were also included in our study to ensure that the data on CYTOR expression in CRC were not missed.

\section{Statistical analysis}

Data were presented as the mean \pm standard deviation. The statistical analyses were conducted using the programs GraphPad (GraphPad Software, San Diego, CA, USA) and SPSS 20.0 (SPSS Inc., Chicago, IL, US), and $p<0.05$ was considered statistically significant. Student's $\mathrm{t}$-test was used to test for significant differences between groups. Two-tailed tests were applied to all data if not specified. Kaplan-Meier survival and correlation analysis were performed in SPSS 20.0.

\section{Experimental procedures}

CRC cell lines were cultured and transfected with either CYTOR shRNA or overexpression vectors. The proliferation and metastasis capacity of the cells were assessed by CCK-8, colony assays and transwell migration/invasion assays in vitro and by subcutaneous tumor growth in nude mice or mouse tail-vein injection assays in vivo. A revised ChIRP protocol and mass spectrometry (MS) were used to pull down and identify CYTOR RNA-binding proteins. RNA immunoprecipitation (RIP) was carried out to verify the interaction between CYTOR and its binding proteins. CYTOR EXON-1-deleted and EXON-4-deleted cells were constructed by combining CRISPR/Cas9 and the donor vector. Co-immunoprecipitation and RIP were performed to confirm the interaction motif of CYTOR. A luciferase reporter system and an immunoblotting assay were used to investigate the downstream regulatory targets and interacting proteins of CYTOR.

\section{Additional methods}

All further information can be found in the Additional file 1 section.

\section{Results}

Overexpression of CYTOR in CRC is associated with poor prognosis

Our previous expression profile data [11] showed that CYTOR was up-regulated in CRC and tumor budding cells (Fig. 1a). We further examined the expression of CYTOR in 138 pairs of matched CRC and normal tissues by qRT-PCR, which revealed that CYTOR was up-regulated in CRC tissues (Fig. 1b). Moreover, there was a significant correlation between CYTOR expression and overall patient survival, and patients with higher CYTOR expression showed worse clinical outcome (Fig. 1c). To validate our results, we evaluated the expression levels of CYTOR in the matched pairs of CRC and normal tissue samples using the TCGA RNA-seq database (Fig. 1d), GEO GDS2947 database (Fig. 1g), GEO GSE31737 database (Fig. 1h), GEO 

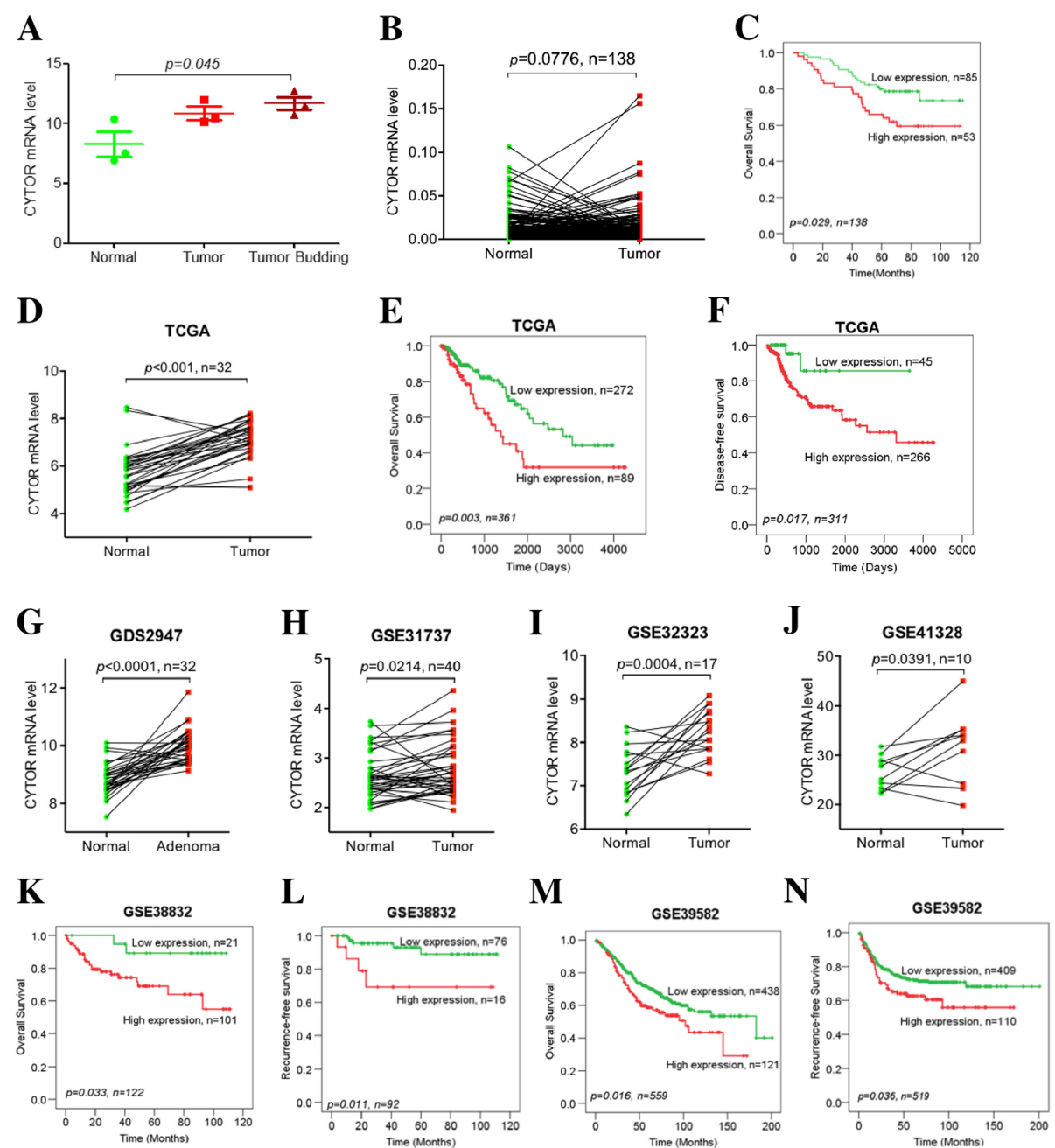

Fig. 1 CYTOR up-regulation in CRC samples with poor outcome. a Up-regulation of CYTOR in laser-microdissection-captured tumor budding cells, tumor cells compared with normal epithelial cells, $n=3$ (CYTOR level represents the fluorescence signal value from microarray). $\mathbf{b}$ Higher expression of CYTOR in CRC samples than the matched normal tissues from our tissue bank, measured by qRT-PCR. c Kaplan-Meier plots of overall survival for CRC samples from our tissue bank, higher CYTOR expression with poorer survival. $\mathbf{d}$ Higher expression of CYTOR in CRC samples than the matched normal tissues from the TCGA database. e Kaplan-Meier plots of overall survival and $\mathbf{f}$ disease-free survival for CRC samples from the TCGA database, higher CYTOR expression with poorer survival. $\mathbf{g}$ Higher expression of CYTOR in CRC samples than the matched normal tissues from the GDS2947, h GSE31737, i GSE32323 and j GSE41328 databases. k Kaplan-Meier plots of overall survival and I recurrence-free survival for CRC samples from the GSE38832 database, higher CYTOR expression with poorer survival. $\mathbf{m}$ Kaplan-Meier plots of overall survival and n recurrence-free survival for CRC samples from the GSE39582 database, higher CYTOR expression with poorer survival

GSE32323 database (Fig. 1i) and GEO GSE41328 database (Fig. 1j). Consistent with our observation, the results from these datasets showed significantly up-regulated CYTOR expression in tumor tissue samples. More interestingly, overall survival data showed a poorer prognosis with higher CYTOR levels in the TCGA database (Fig. 1e), GEO GSE38832 database (Fig. 1k), GEO GSE39582 database (Fig. 1m), GEO GSE17536 database (Additional file 2: Figure S1A), GEO GSE17537 database (Additional file 2: Figure S1C), and GEO GSE29621 database (Additional file 2: Figure S1H). Similarly, the expression of CYTOR in CRC was also negatively correlated with disease- or recurrence-free survival in the TCGA database (Fig. 1f),
GEO GSE38832 database (Fig. 11), GEO GSE39582 database (Fig. 1n), GEO GSE17536 database (Additional file 2: Figure S1B), GEO GSE24549-GPL5175 database (Additional file 2: Figure S1J), and GEO GSE24550-GPL11028 database (Additional file 2: Figure S1K).

However, no such correlation was observed between CYTOR and overall, disease-free or recurrence-free survival in some other databases, such as GEO GSE17537 (Additional file 2: Figure S1D), GEO GSE56699 (Additional file 2: Figure S1E and F), GEO GSE16125 (Additional file 2: Figure S1G), GEO GSE24549-GPL11028 (Additional file 2: Figure S1I), GEO GSE24550-GPL5175 (Additional file 2: Figure S1L), GEO GSE31595 (Additional file 2: 
Figure S1M) and GEO GSE33113 (Additional file 2: Figure S1N). To address this issue, we performed a meta-analysis to systematically evaluate the association between CYTOR and CRC survival risk, combining our data with all the other online CYTOR data. When the cutoff value of CYTOR expression was set according to the receiver operating characteristic (ROC) curve, the patients with higher expression of CYTOR had significantly poorer overall survival (OS; pooled HR, 1.86; 95\% CI, 1.50-2.30; Additional file 3: Figure S2A) and disease- or recurrence-free survival (DFS; pooled HR, 1.67, 95\%CI, 1.33-2.09; Additional file 3: Figure S2B). When the cutoff value of CYTOR expression was set as P50, CYTOR expression was also negatively associated with overall survival (OS; pooled $\mathrm{HR}, 1.22$; $95 \% \mathrm{CI}$, 1.01-1.48; Additional file 3: Figure S2C) and diseaseor recurrence-free survival (DFS; pooled HR, 1.30, 95\%CI, 1.07-1.58; Additional file 3: Figure S2D). Funnel plots further showed no bias among these databases, which confirmed that CYTOR was a risk factor for survival in colorectal cancer (Additional file 4: Figure S3). Taken together, these clinical data revealed the strong association between CYTOR expression and CRC development/prognosis.

\section{CYTOR promotes CRC progression in vitro and in mouse xenografts}

Next, we examined the CYTOR levels in CRC cell lines, and higher expression was found in RKO, SW480 and SW620 cells than in HCT116 or HCT8 cells (Fig. 2a). When we stably knocked down CYTOR in RKO, SW480 and SW620 cells (Fig. 2b), the colony-forming potential of RKO and SW620 was inhibited (Fig. 2c), and no colonies of SW480 cells were observed. In addition, shRNA-mediated CYTOR knockdown significantly decreased migration and invasion compared with the scramble control in these cell lines (Fig. 2d). To avoid off-target effects of shRNA, we designed and synthesized another two siRNAs to knockdown CYTOR in RKO, SW480 and SW620 cells (Additional file 5: Figure S4A). The results showed knockdown of CYTOR by siRNAs also inhibited the potential of colony-forming (Additional file 5: Figure S4B), migration and invasion in RKO (Additional file 5: Figure S4C), SW480 (Additional file 5: Figure S4D) and SW620 (Additional file 5: Figure S4E) cell lines, which were consistent with the results from shRNA. Furthermore, ectopic expression of CYTOR in the HCT116 and HCT8 cell lines (Fig. 2e) not only promoted colony formation (Fig. 2f) but also enhanced the capacity for migration and invasion (Fig. 2g). More interestingly, when the ectopic expression of CYTOR was inhibited by specific siRNAs in the transfected HCT116 cells (Fig. 2h), the enhanced migration and invasion were also decreased by these siRNAs (Fig. 2i).
These results demonstrated that CYTOR could promote the anchorage-independent growth, migration and invasion of CRC cells in vitro. As tumor budding cells are considered a histological phenomenon of EMT, which contributes to tumor metastasis [19-21], we also evaluated the relationship between CYTOR and EMT markers. In SW480 and SW620 cells, knockdown of CYTOR increased E-cadherin expression while decreasing Vimentin expression (Fig. 2j). On the other hand, overexpression of CYTOR inhibited E-cadherin expression while increasing Vimentin expression in HCT8 and HCT116 cells (Fig. 2k). Analysis of the current GEO database (GSE29621 and GSE38832) showed that CYTOR expression was negatively correlated with the epithelial marker E-cadherin and positively correlated with mesenchymal markers including Vimentin, $\mathrm{N}$-cadherin, FN1, Twist, and MMP9 (Additional file 6: Figure S5).

We also investigated whether CYTOR was functionally involved in CRC progression in mouse xenografts. When RKO cells transfected with CYTOR shRNA were subcutaneously injected into nude mice, the volume (Fig. 3a) and weight (Fig. 3b) of the xenograft tumors were significantly decreased compared with the scramble control group. Moreover, tumor growth was also repressed by CYTOR knockdown as shown in the tumor growth curve (Fig. 3c). By contrast, overexpression of CYTOR increased the volume (Fig. 3d) and weight (Fig. 3e) of the xenograft tumors and promoted tumor growth in vivo (Fig. 3f). To explore whether CYTOR also promotes CRC metastasis in vivo, we intravenously injected luciferase-labeled control or CYTOR knockdown RKO cells into NOD/SCID mice and subjected them to bioluminescent imaging to monitor metastasis. The results showed that the whole-body luminescence signals in the CYTOR knockdown group were significantly reduced compared with those of the control group after 30 days (Fig. 3g). Overall, our data indicated that CYTOR could promote EMT and CRC progression.

\section{CYTOR mediates complex formation between NCL and Sam68}

To explore the detailed mechanism whereby CYTOR regulates CRC progression, we first investigated the distribution of CTYOR in RKO cells and found it distributed in both cytoplasm and nucleus (Additional file 7: Figure S6A). Then, we designed a set of specific probes labeled with biotin to pull down the proteins that directly bind to CYTOR $[8,22]$. The efficiency and specificity of the probes were confirmed by PCR (Fig. 4a). We isolated the proteins from the pull-down complex by SDS-PAGE electrophoresis (Additional file 7: Figure S6B) and identified NCL and Sam68 as the CYTOR-binding proteins by MS assays (Additional file 7: Figure S6C) in RKO cells. Furthermore, the interactions between CYTOR and NCL, 

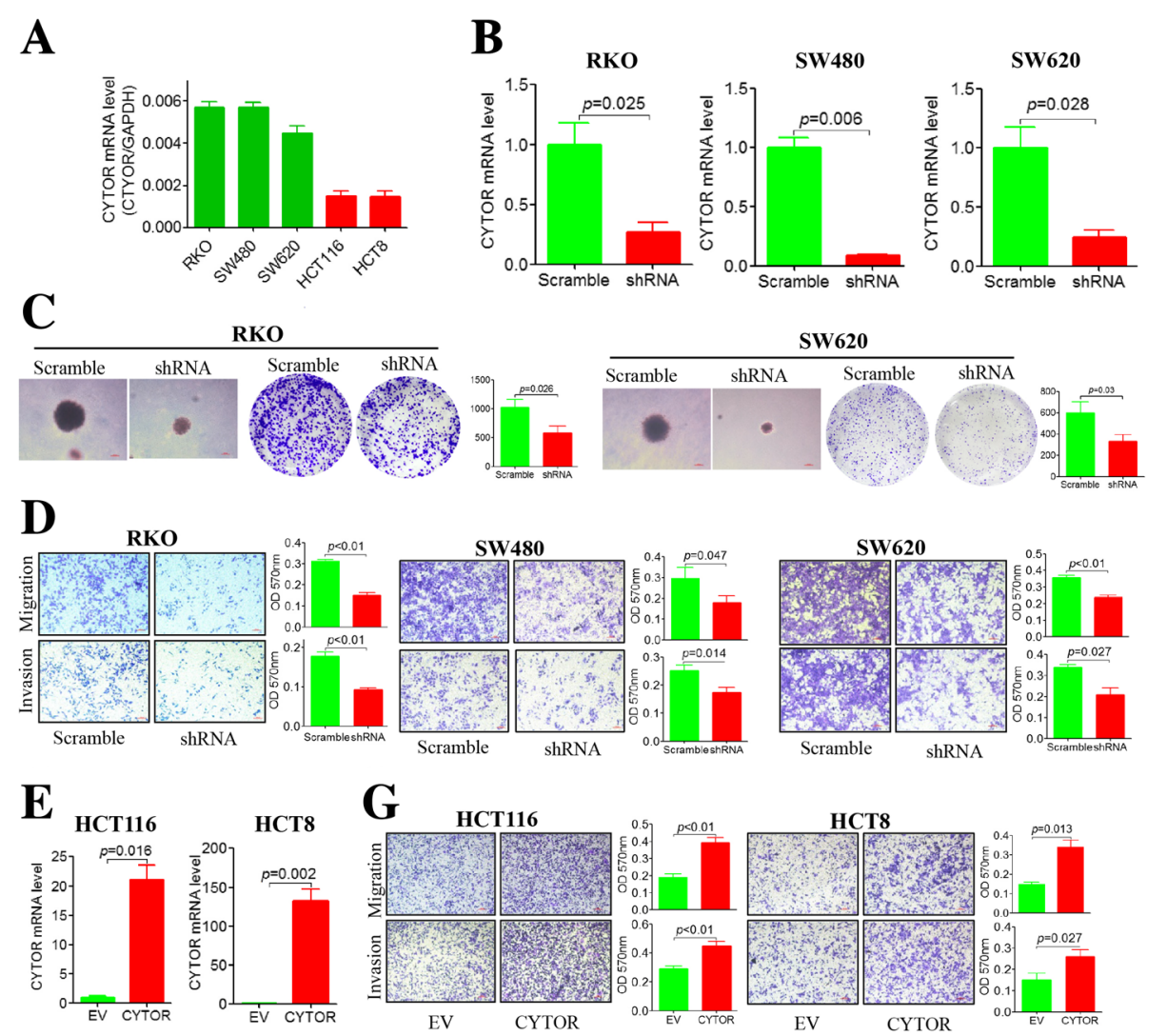

G
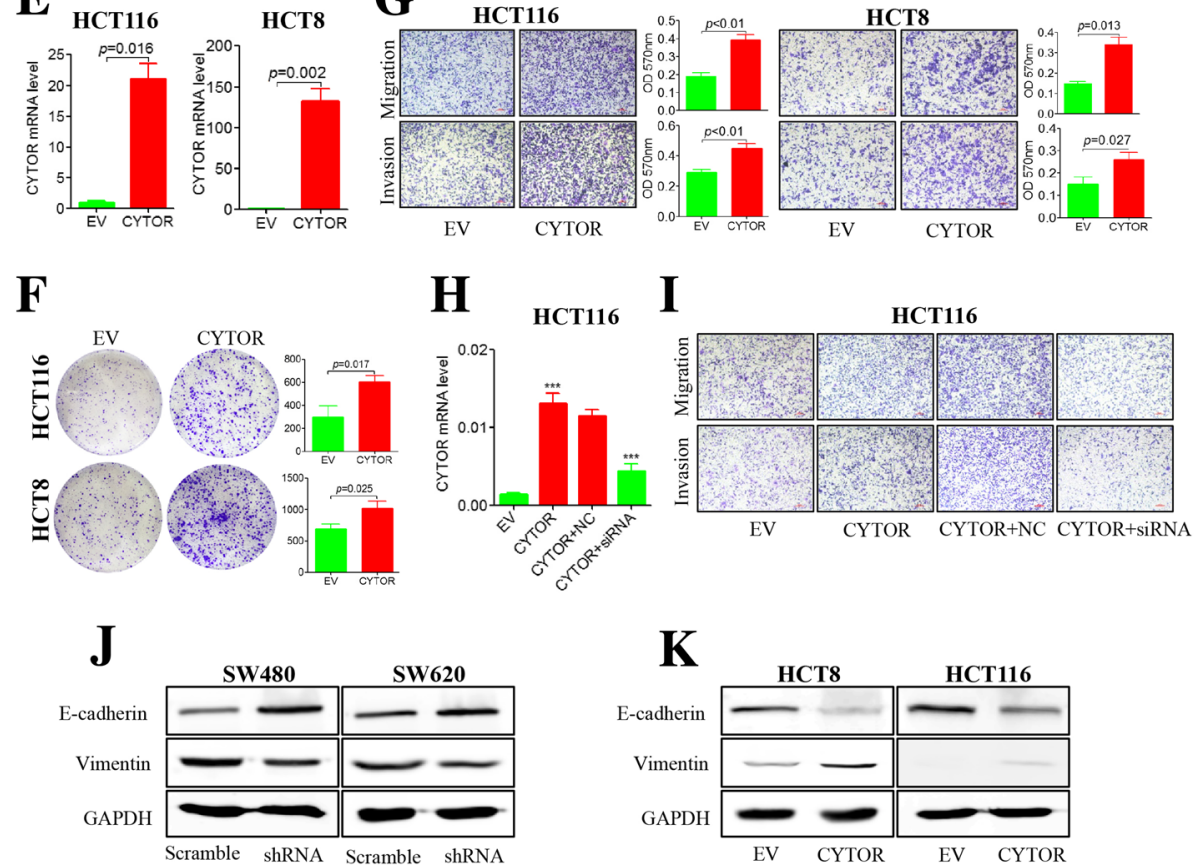

Fig. 2 CYTOR promotes anchorage-independent growth and migration/invasion in vitro. a CYTOR levels in RKO, SW480, SW620, HCT116 and HCT8 cell lines by qRT-PCR. b Knockdown of CYTOR by shRNA in the RKO, SW480 and SW620 cell lines. c Reduction of colony formation ability for CYTOR knockdown (shRNA) RKO and SW620 cells compared with control (scramble) by soft agar and plates assay (right histogram represents quantification analysis). $\mathbf{d}$ Decrease of migration/invasive potential for CYTOR knockdown RKO, SW480 and SW620 cells compared with control by transwell assay (right histogram represents quantification analysis). e qRT-PCR for CYTOR levels in empty-vector (EV) and overexpression-vector (CYTOR)-transfected HCT116 and HCT8 cells. $\mathbf{f}$ Increase of colony formation ability for overexpression-vector (CYTOR)- transfected HCT116 and HCT8 cells compared with empty-vector (EV) by plates colony formation assay (Right histogram represents quantification analysis). $\mathbf{g}$ Increase of migration/invasive potential for CYTOR overexpression (CYTOR) HCT116 and HCT8 cells compared with empty-vector (EV) by transwell assay (Right histogram represents quantification analysis). $\mathbf{h}$ qRT-PCR for CYTOR levels in HCT116 cells with a CYTOR overexpression vector (CYTOR) and co-transfection CYTOR siRNA (CYTOR+siRNA). i Increase of migration/invasive potential for CYTOR-overexpressing (CYTOR, Lane 2) HCT116 cells and rescue potential of CYTOR siRNA (CYTOR+siRNA, Lane 4) by transwell assay. $\mathbf{j}$ Expression of E-cadherin and Vimentin in CYTOR knockdown SW480 and SW620 cells by immunoblotting. $\mathbf{k}$ Expression of E-cadherin and Vimentin in CYTOR-overexpressing HCT116 and HCT8 cells by immunoblotting 


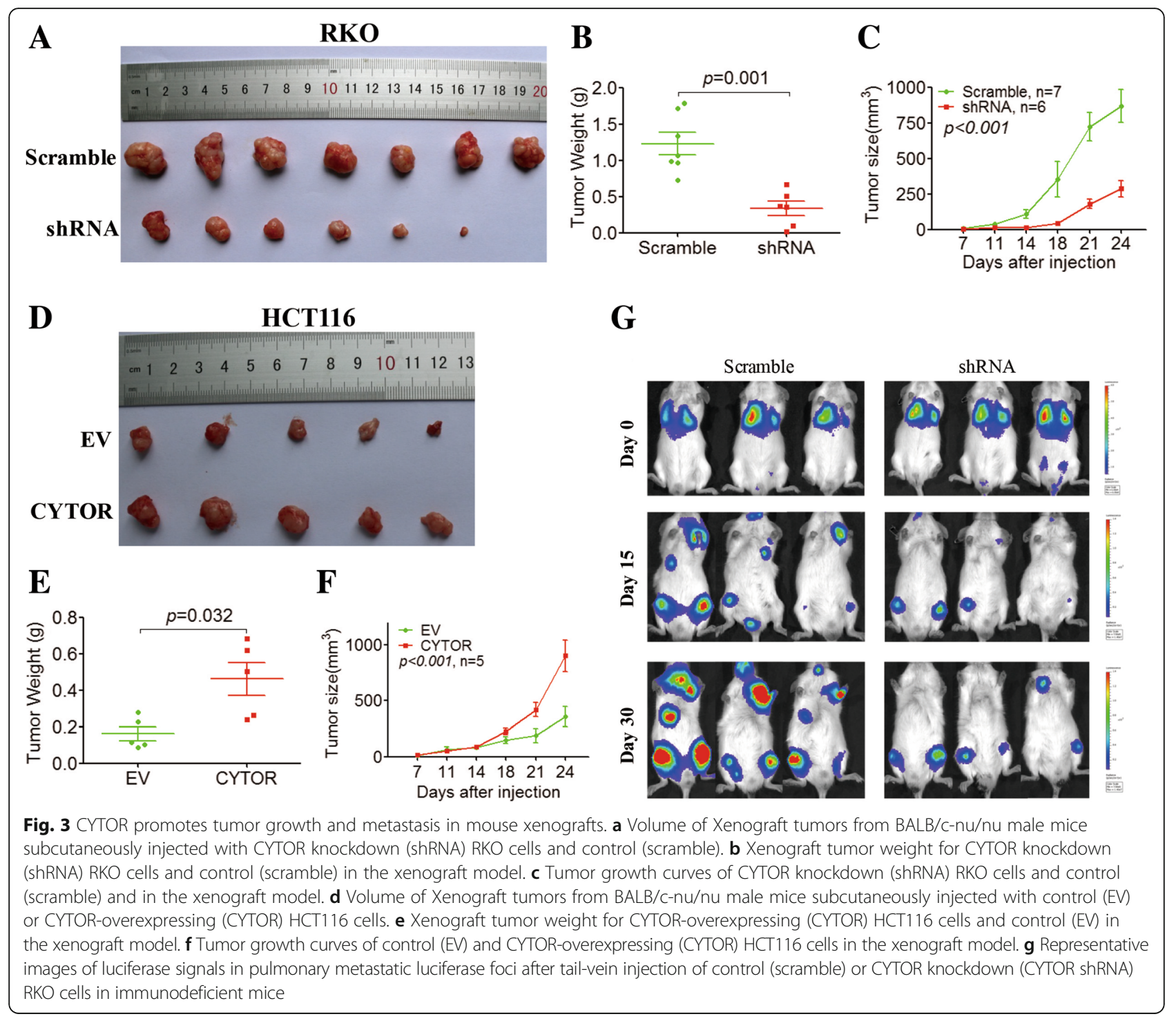

CYTOR and Sam68 were confirmed by immunoblotting (Fig. 4b). In addition, RIP assays revealed that both NCL (Fig. 4c) and Sam68 (Fig. 4d) could pull down CYTOR directly.

The results above demonstrated that NCL and Sam68 could bind to CYTOR; next, we investigated whether a complex could be formed between NCL and Sam68. The co-IP assay showed that NCL was able not only to pull down Sam68 (Fig. 4e) but also to be immunoprecipitated by Sam68 (Fig. 4f) in live cells. Then, we simultaneously transfected the FLAG-tagged NCL (NCL-FLAG) and HA-tagged Sam68 (Sam68-HA) expression vectors into $293 \mathrm{~T}$ cells, and the harvested cell lysates were treated with either RNase inhibitor or RNaseA, followed by dual co-IP assays with either anti-FLAG or anti-HA antibody. The results showed that NCL and Sam68 protein could be co-immunoprecipitated reciprocally in the RNaseinhibitor-treated samples (Fig. $4 \mathrm{~g}$ and h, 3rd lanes) but not in RNaseA-treated samples (Fig. 4g and h, 6th lanes). These results suggested that the interaction between NCL and Sam68 depended on the existence of RNA.

The NCL protein consists of six main domains, including the N-terminal domain, four RNA binding domains (RBDs) and the GAR domain [23]. To further identify the sites on NCL that interact with CYTOR, we constructed four deletion mutants of FLAG-tagged NCL (Fig. 4i). It was shown that in addition to full-length NCL (FL), the mutants that included the 3rd and 4th RBDs and the GAR domain could pull down CYTOR (Fig. 4j), indicating that these domains might be the key sites of interaction with CYTOR. Moreover, we also constructed $\mathrm{KH}$-domain-deleted $(\Delta \mathrm{KH}), \mathrm{N}$-terminus-deleted $(\Delta \mathrm{N})$ and C-terminus-deleted $(\Delta \mathrm{C})$ HA-tagged Sam68 expression vectors [24] (Fig. 4k). RIP results revealed that the $\Delta \mathrm{C}$ mutant could bind CYTOR, while the $\Delta \mathrm{KH}$ and $\Delta \mathrm{N}$ mutants could not (Fig. 4l), demonstrating that 


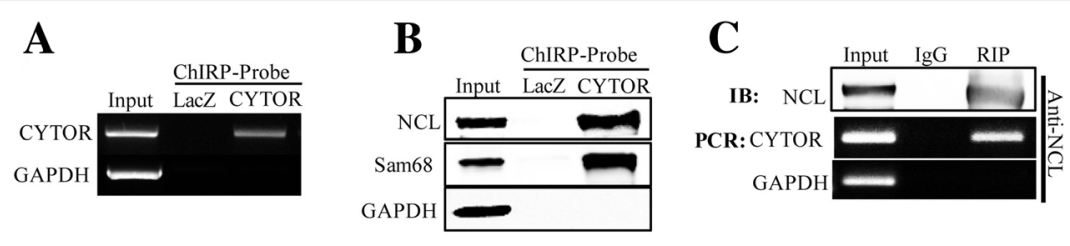

D

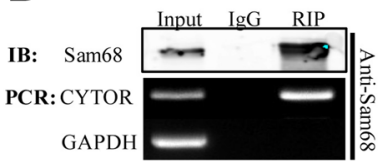

G

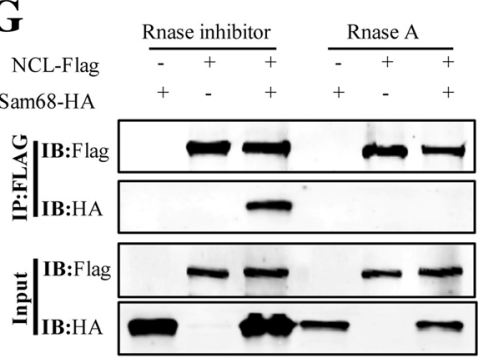

I

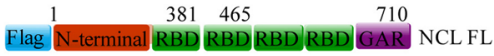
Flag N-terminal RBD

Flag RBD RBD RBD GAR 382-710 Flag N-terminal RBD RBD Flag RBD RBD GAR 466-710

$\mathbf{J}$

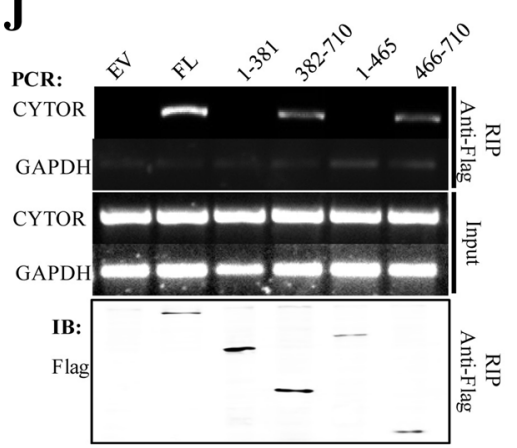

M

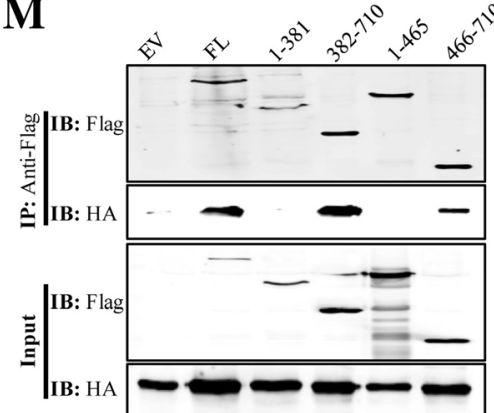

$\mathbf{F}$

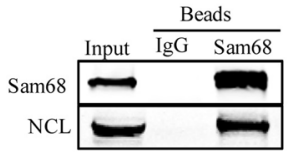

H

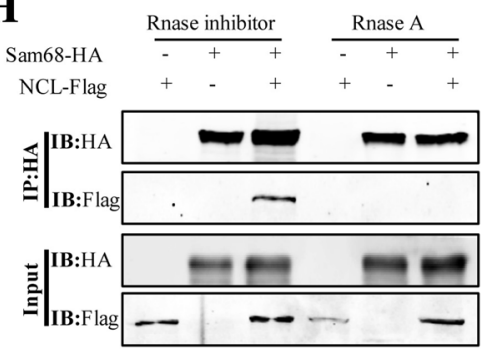

$\mathbf{K}$

$\mathrm{N}$-terminal $\square \mathrm{KH} \square \mathrm{C}$-terminal $\mathrm{HA}$ Sam68 FL N-terminal $=$ C-terminal HA $\mathrm{HA}$ $\mathrm{N}$-terminal $\mathrm{KH}=\mathrm{HA}$

$\Delta \mathrm{C}$

EKHE C-terminal $\mathrm{HA} \Delta \mathrm{N}$

$\mathbf{L}$

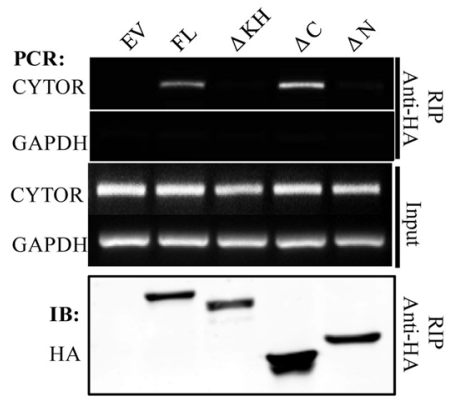

$\mathbf{N}$

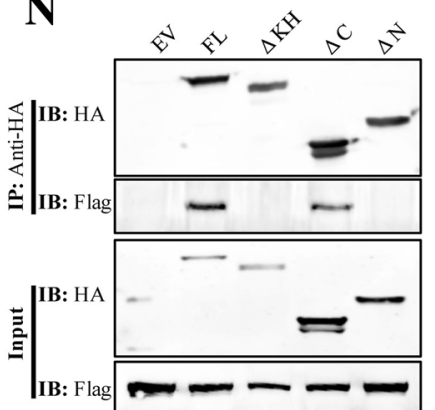

Fig. 4 (See legend on next page.) 


\begin{abstract}
(See figure on previous page.)
Fig. 4 CYTOR mediates complex formation between NCL and Sam68. a RT-PCR for detection of CYTOR and GAPDH pull down by ChIRP probes of LacZ and CYTOR in RKO cells. b Immunoblotting for detection of NCL and Sam68 pull down by ChIRP probes of LacZ and CYTOR in RKO cells. c Immunoblotting for detection of NCL (upper panel) and RT-PCR for detection of CYTOR and GAPDH (lower panels) by RIP with antibody against NCL in RKO cells. $\mathbf{d}$ Immunoblotting for detection of Sam68 (upper panel) and RT-PCR for detection of CYTOR and GAPDH (lower panels) by RIP with antibody against Sam68 in RKO cells. e Immunoblotting for detection of endogenous NCL and Sam68 immunoprecipitated by NCL-specific antibody in RKO cells. $\mathbf{f}$ Immunoblotting for detection of endogenous Sam68 and NCL immunoprecipitated by Sam68-specific antibody in RKO cells. $\mathbf{g}$ Immunoblotting for detection of exogenous FLAG-tagged NCL and HA-tagged Sam68 immunoprecipitated by anti-FLAG antibody with or without RNaseA treatment in 293 T cells. h Immunoblotting for detection of exogenous HA-tagged Sam68 and FLAG-tagged NCL immunoprecipitated by anti-HA antibody with or without RNaseA treatment in 293 T cells. i Schematic of the FLAG-tagged full-length and truncation mutant constructs of NCL. j RT-PCR for detection of CYTOR and GAPDH (upper panel) and immunoblotting for detection of truncation mutant NCL with anti-FLAG (lower panels) by RIP with anti-FLAG antibody in 293 T cells with different truncation mutant constructs of NCL. k Schematic of the HA-tagged full-length and truncation mutant constructs of Sam68. I RT-PCR for detection of CYTOR and GAPDH (upper panel) and immunoblotting for detection of truncation mutant Sam68 with anti-HA (lower panels) by RIP with anti-HA antibody in 293 T cells with different truncation mutant constructs of Sam68. $\mathbf{m}$ Immunoblotting for detection of exogenous HA-tagged Sam68 and different FLAG-tagged truncation mutants of NCL immunoprecipitated by anti-FLAG antibody in 293 T cells. $\mathbf{n}$ Immunoblotting for detection of exogenous FLAG-tagged $\mathrm{NCL}$ and different HA-tagged truncation mutants of Sam68 immunoprecipitated by by anti-HA antibody in $293 \mathrm{~T}$ cells
\end{abstract}

the $\mathrm{KH}$ and $\mathrm{N}$-terminal domains were the sites of interaction with CYTOR. More intriguingly, co-IP assays revealed that the 3rd and 4th RBDs and the GAR domain of NCL were required for binding to Sam68 (Fig. $4 \mathrm{~m}$ ), and the $\mathrm{KH}$ and $\mathrm{N}$-terminal domains of Sam68 were indispensable to its interaction with NCL (Fig. 4n). Taken together, the above data indicated that CYTOR plays an essential part in forming a complex between NCL and Sam68 by interacting with specific motifs on the two proteins.

\section{EXON1 of CYTOR is important for its biological function of forming a complex with NCL and Sam68}

We next intended to identify the motifs in CYTOR that are essential for its interaction with NCL and Sam68. The secondary structure of CYTOR was predicted using the RNA fold web server [25]. It was found that only the EXON1 deletion $(\triangle \mathrm{EXON} 1)$ and not the other EXON deletion mutants $(\triangle \mathrm{EXON} 2, \triangle \mathrm{EXON} 3$ and $\triangle \mathrm{EXON4)}$ of CYTOR could induce a dramatic change in the secondary structure relative to wild-type (WT) CYTOR (Fig. 5a). Thus, we used the CRISPR/Cas9 method (Fig. 5b) to construct $\triangle$ EXON1 (Fig. 5c) and $\triangle$ EXON4 RKO cells (Fig. 5d). The mutant cells were screened and confirmed as homozygous EXON1-deleted (Fig. 5e) and EXON4-deleted (Fig. 5f) clones by sequencing. As shown in Fig. 6a, the colony-forming potential of $\triangle$ EXON1 cells was reduced, but no changes were observed in $\triangle$ EXON4 cells. Similarly, deleting EXON1 of CYTOR inhibited cell migration and invasion, but deleting EXON4 had no such effect (Fig. 6b). Interestingly, a ChIRP assay showed that CYTOR-specific probes could pull down NCL and Sam68 in wild-type and $\triangle \mathrm{EXON4}$ RKO cells but not in $\triangle \mathrm{EXON1}$ RKO cells (Fig. 6c). Additionally, an RIP assay was performed to investigate the endogenous interactions between NCL, Sam68 and CYTOR in these mutant cells, and the results showed that while both NCL and Sam68 could bind to CYTOR in $\triangle$ EXON4 RKO cells, neither could pull down CYTOR in $\triangle$ EXON1 RKO cells (Fig. 6d). To determine whether complex formation between NCL and Sam68 also requires EXON1 of CYTOR, we evaluated their interaction in $\triangle \mathrm{EXON1}$ and $\triangle \mathrm{EXON4}$ cells using co-IP. The results showed that while NCL and Sam68 could be immunoprecipitated reciprocally in $\triangle$ EXON4 cells (Fig. 6f), no such interaction could be observed in $\triangle \mathrm{EXON} 1$ cells (Fig. 6e).

To further verify the above results, we co-transfected $293 \mathrm{~T}$ cells with exogenous expression vectors for FLAG-tagged NCL, CYTOR EXON1-deletion (CYTOR$\Delta 1)$ or EXON4-deletion (CYTOR- $\Delta 4$ ) mutants. An RIP assay with anti-FLAG antibody revealed that NCL could bind to CYTOR- $\Delta 4$ but not CYTOR- $\Delta 1$ (Fig. $6 g$ ). Similarly, HA-tagged Sam 68 could also pull down CYTOR- $\Delta 4$ but not CYTOR- $\Delta 1$ (Fig. 6h). Fortunately, we found two NCL-specific binding motifs [26] at the 125th nt of EXON1 and the 602nd nt of EXON4 in the CYTOR RNA sequence, as well as two Sam68-specific binding motifs [27] at the 60th nt of EXON1 and the 786th nt of EXON4 (Fig. 6i). Therefore, we constructed a series of CYTOR expression vectors with mutations in these sites (Fig. 6i). When FLAG-tagged NCL and CYTOR mutants with mutations in the NCL-specific sites (CYTOR-m1 for mutation in EXON1 or CYTOR-m2 for mutation in EXON4) were co-transfected into 293 T cells, an RIP assay showed that NCL could bind to CYTOR-m2 but not to CYTOR-m1 (Fig. 6j). Similarly, while HA-tagged Sam68 could not pull down the CYTOR mutant with the mutation in its specific-binding site on EXON1 (CYTOR-m3), it could bind to the CYTOR mutant with the mutation on EXON4 (CYTOR-m4) (Fig. 6k). Taken together, these data indicated that the formation of heterotrimeric complex of NCL, Sam68, and CYTOR requires their specific interacting sites in CYTOR EXON1. 

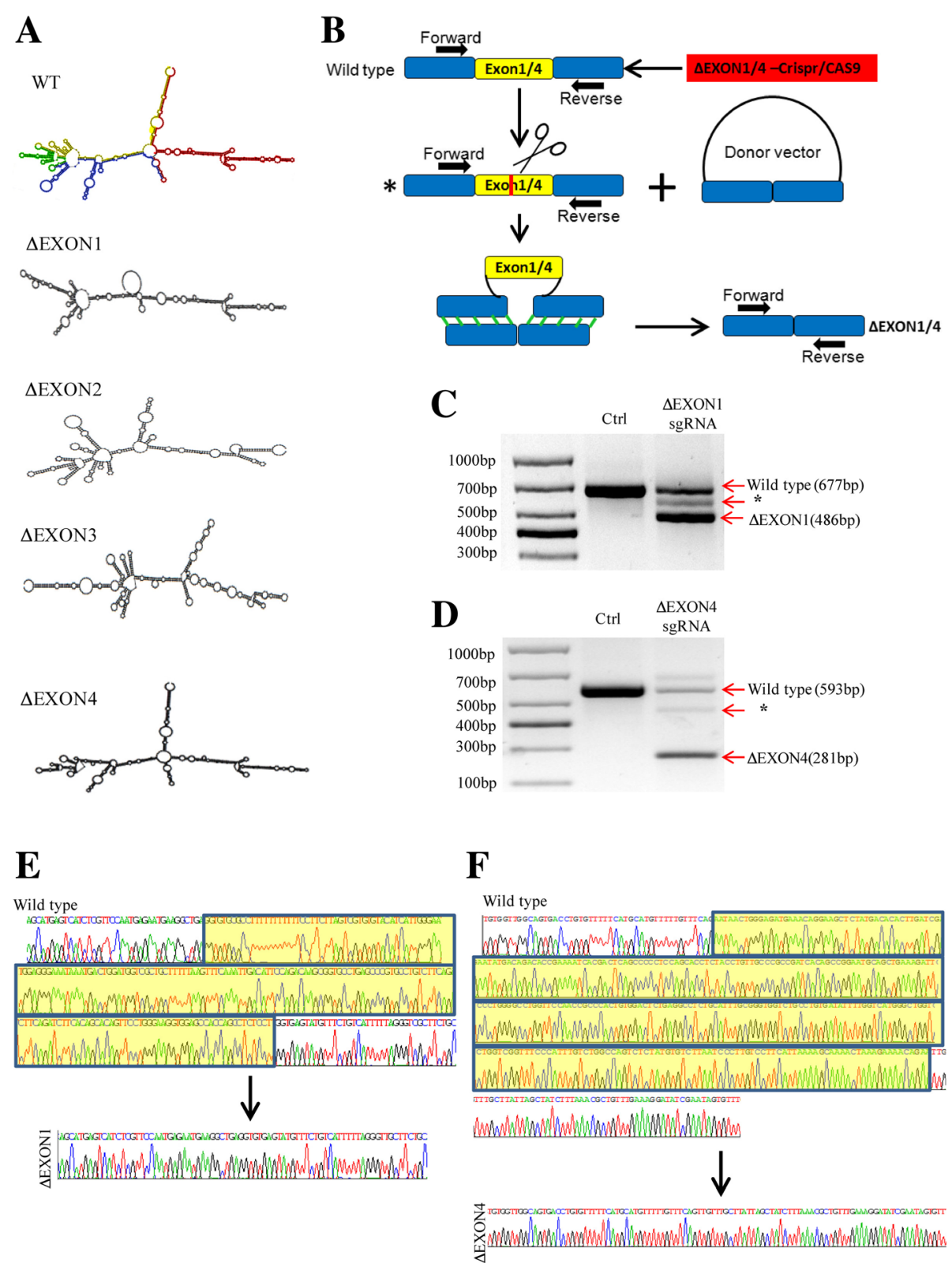

Fig. 5 Production of CYTOR mutant cells by CRISPR/Cas9. a Prediction of secondary RNA structure of wild-type (WT) CYTOR and various exon-deleted CYTOR mutants. b Schematic diagram for CRISPR/Cas9 and donor vector to delete EXON1 or EXON4 of CYTOR. c RT-PCR and gel electrophoresis to assess the editing efficiency of CRISPR-sgRNA specific to EXON 1. *indicates Non-homologous end joining with an Indel. $\mathbf{d}$ RT-PCR and gel electrophoresis to assess the editing efficiency of CRISPR-sgRNA specific to EXON 4. *indicates Non-homologous end joining with an Indel. e DNA sequencing to identify the exon1-deleted RKO cells ( $\triangle E X O N 1$ ) through clone screening. $\mathbf{f}$ DNA sequencing to identify the exon4-deleted RKO cells $(\triangle \mathrm{EXON4)}$ through clone screening

\section{$\mathrm{NCL}$ and Sam68 act as oncogenes to promote CRC progression}

Although it was confirmed that NCL and Sam68 could directly interact with CYTOR to form a complex, their function in CRC progression was still unknown. The results from online databases showed that NCL (Fig. 7a and Additional file 8: Figure S7A) and Sam68 (Fig. 7b and Additional file 8: Figure S7B) were both up-regulated in tumor samples compared with paired normal samples. In addition, the expression levels of CYTOR, NCL, and Sam68 were positively correlated (Fig. 7c). Our IHC results also revealed that higher NCL (Fig. 7d) and Sam68 (Fig. 7e) expression levels were associated with poorer survival rates. These results suggest that both NCL and Sam68 are involved in CRC progression. 

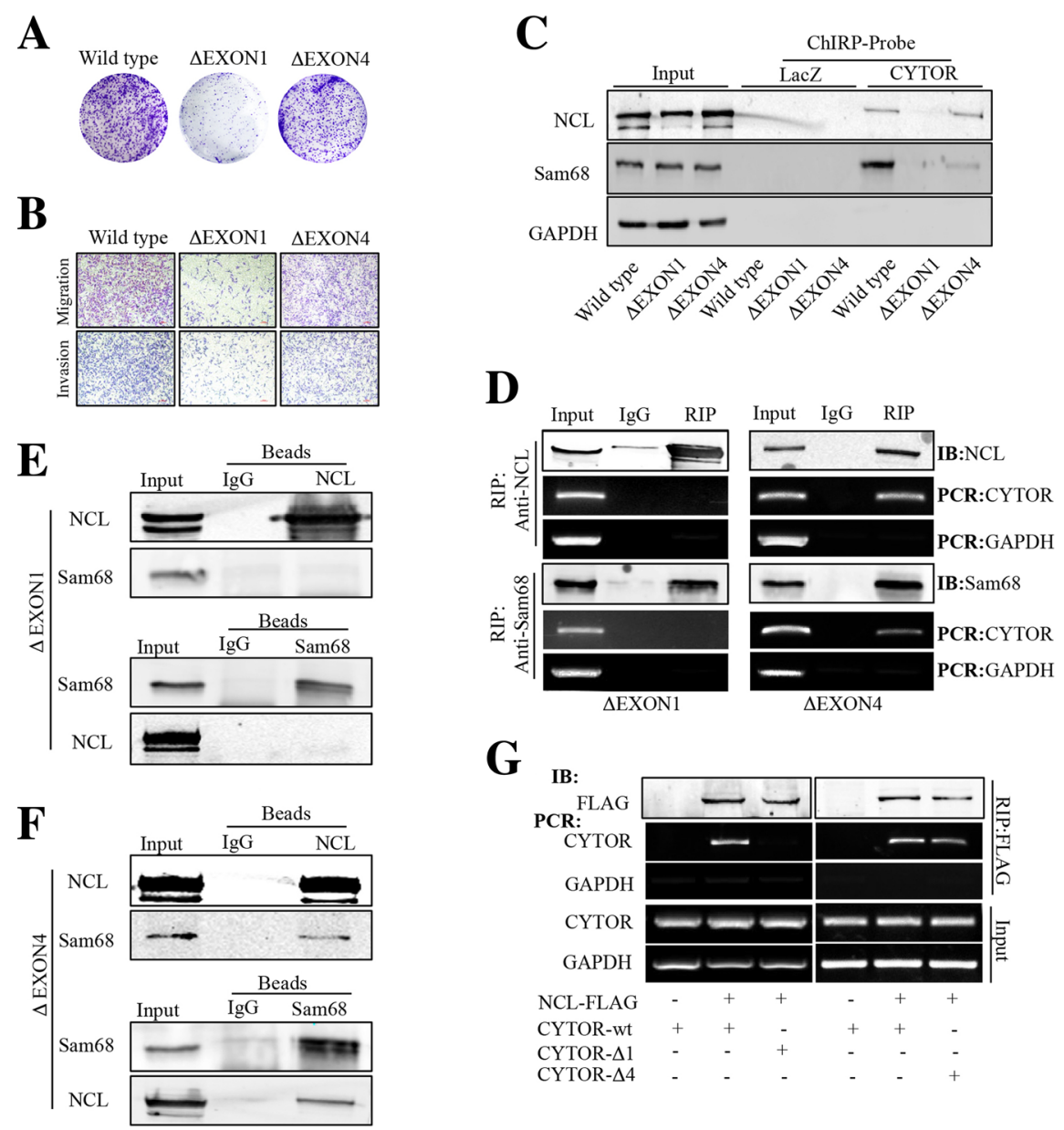

G
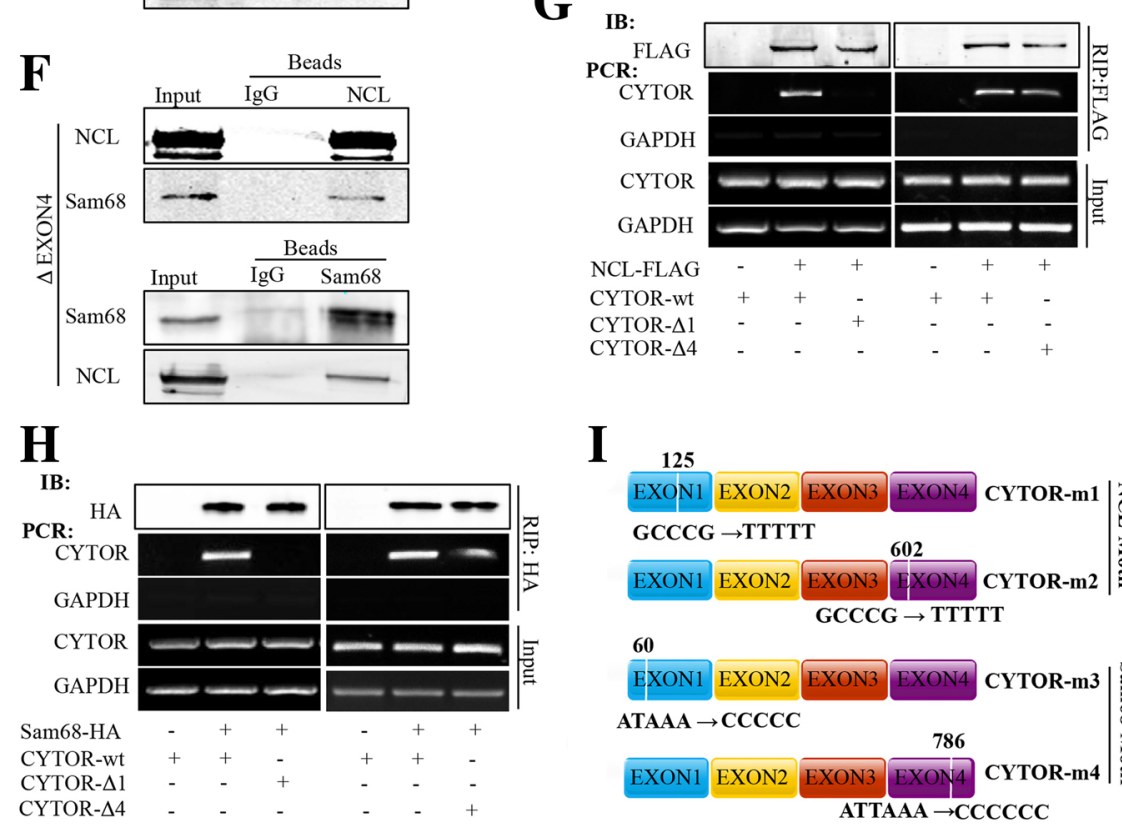

I

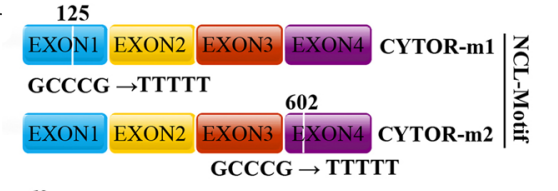
60

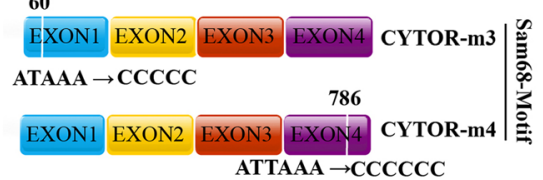

J $\quad$ K

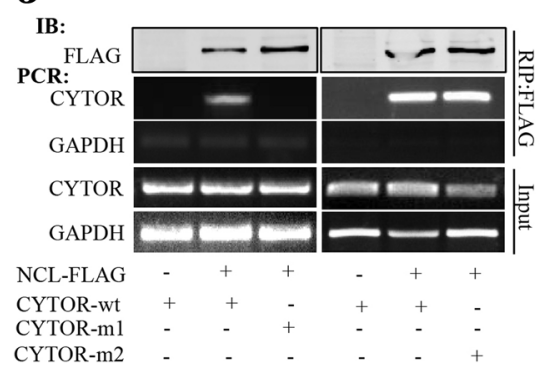

K

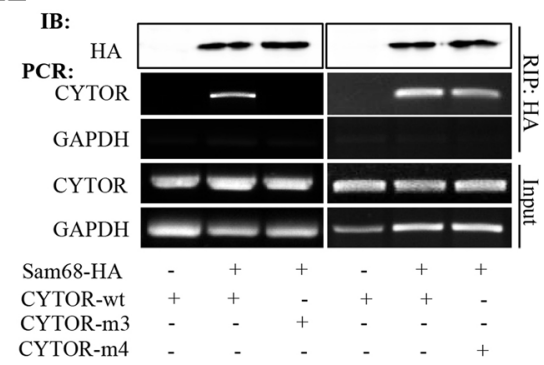

Fig. 6 (See legend on next page.) 


\begin{abstract}
(See figure on previous page.)
Fig. 6 EXON1 is a key functional site of CYTOR and mediates the interaction between NCL and Sam68. a Decrease of colony formation ability for EXON1-deleted mutant ( $\triangle$ EXON1) and EXON4-deleted mutant ( $\triangle$ EXON4) RKO cells by CRISPR/Cas9 compared with wild-type. $\mathbf{b}$ Decrease of migration/invasive potential for EXON1-deleted mutant ( $\triangle E X O N 1)$ and EXON4-deleted mutant ( $\triangle E X O N 4)$ RKO cells compared with wild-type. c Immunoblotting for detection of NCL and Sam68 pull down by ChIRP probes of LacZ and CYTOR in wild-type, EXON1-deleted mutant $(\triangle E X O N 1)$ and EXON4-deleted mutant ( $\triangle E X O N 4)$ RKO cells. $\mathbf{d}$ Immunoblotting for detection of NCL and Sam68 and RT-PCR for detection of CYTOR and GAPDH by RIP with antibodies against NCL and Sam68 in EXON1-deleted mutant ( $\triangle E X O N 1)$ and EXON4-deleted mutant ( $\triangle E X O N 4)$ RKO cells. e Reciprocal immunoprecipitation between endogenous Sam68 and NCL with separate specific antibodies in EXON1-deleted mutant $(\triangle E X O N 1)$ RKO cells. $\mathbf{f}$ Reciprocal immunoprecipitation between endogenous Sam68 and NCL with separate specific antibodies in EXON4-deleted mutant ( $\triangle E$ EXON4) RKO cells. $\mathbf{g}$ RT-PCR for detection of CYTOR and GAPDH by RIP with anti-FLAG in 293 T cells co-transfected with FLAG-tagged NCL and wild-type (CYTOR-wt), EXON1-deleted mutant (CYTOR- $\Delta 1$ ) or EXON4-deleted mutant (CYTOR- $\Delta 4$ ) CYTOR. $\mathbf{h}$ RT-PCR for detection of CYTOR and GAPDH by RIP with anti-HA in 293 T cells co-transfected with HA-tagged Sam68 and wild-type, EXON1-deleted mutant or EXON4-deleted mutant CYTOR. i Schematic of CYTOR motif mutant constructs; CYTOR-m1, mutant for the NCL-specific motif in EXON1 of CYTOR; CYTOR-m2, mutant for the NCL-specific motif in EXON4 of CYTOR; CYTOR-m3, mutant for the Sam68-specific motif in EXON1 of CYTOR; CYTOR-m4, mutant for the Sam68-specific motif of Sam68 in EXON4 of CYTOR. $\mathbf{j}$ RT-PCR for detection of CYTOR and GAPDH by RIP with anti-FLAG in 293 T cells co-transfected with FLAG-tagged NCL and wild-type CYTOR, CYTOR-m1 and CYTOR-m2. k RT-PCR for detection of CYTOR and GAPDH by RIP with anti-HA in 293 T cells co-transfected with HA-tagged Sam68 and wild-type CYTOR, CYTOR-m3 and CYTOR-m4
\end{abstract}

In an effort to decipher the detailed functions of both proteins, we knocked down NCL or Sam68 using siRNAs (Fig. 7f), and it was shown that the proliferation of SW620 (Fig. 7g) and RKO (Additional file 8: Figure S7C) cells was inhibited under those conditions. Consistently with the CYTOR knockdown results, loss of NCL and Sam68 also repressed the migration and invasion activity of SW620 (Fig. 7h) and RKO (Additional file 8: Figure S7D) cells. Furthermore, the epithelial marker E-cadherin was increased and the mesenchymal marker Vimentin was decreased by NCL and Sam68 siRNAs (Fig. 7i). To verify these observations, we investigated the relationship between the expression of NCL/Sam68 and EMT markers in the GSE38832 database, and the results showed that both NCL and Sam68 expression were negatively correlated with the epithelial marker E-cadherin and positively correlated with the mesenchymal marker Vimentin, $\mathrm{N}$-cadherin, Snail, and Twist (Additional file 9: Figure S8A for NCL and Additional file 9: Figure S8B for Sam68). Collectively, NCL and Sam68 may function as oncogenes to promote CRC EMT and progression.

\section{The NCL-CYTOR-Sam68 complex activates the NF-KB pathway}

The NF-kB pathway has been recognized as a key player in CRC progression, being involved in the EMT process $[28,29]$; therefore, we evaluated the effect of the CYTOR-NCL-Sam68 complex on the NF-kB signaling pathway. The results showed that both NCL and Sam68 knockdown caused a dramatic decrease in phosphorylated P65 in CRC cell lines (Fig. 8a) and attenuated the transcriptional activity of $\mathrm{NF}-\mathrm{kB}$ as shown by a luciferase promoter assay (Fig. 8b). Similarly, CYTOR knockdown also significantly decreased the level of phosphorylated P65, while overexpression of CYTOR increased the expression of phosphorylated P65 (Fig. 8c). Moreover, a luciferase promoter assay showed decreased NF-kB promoter activity when CYTOR expression was inhibited by shRNA (Fig. 8d). These results demonstrated that the NCL-CYTOR-Sam68 complex might promote CRC progression by activating the NF- $\mathrm{kB}$ signaling pathway.

\section{The NCL-CYTOR-Sam68 complex can be used as a biomarker for CRC prognosis}

To further evaluate the clinical significance of the CYTOR-NCL-Sam68 complex, we performed ROC analysis for CYTOR, NCL and Sam68, either separately or combined, in the TCGA, GSE17536 and GSE17537 databases. The results showed that the AUC for combined CYTOR, NCL, and Sam68 was greater than the individual AUC for CYTOR, NCL or Sam68 (Fig. 8e). Interestingly, in the $3 \mathrm{D}$ curve assay, the area with high expression of CYTOR, NCL and Sam68 represented samples from recurrent patients (Fig. 8f). The overall survival data from the GSE17536 database also showed that the patients with high expression of all three molecules (group 4) displayed the worst prognosis (Fig. 8g). These clinical data suggest that the NCL-CYTOR-Sam68 complex can be used as a biomarker for CRC recurrence and prognosis.

\section{Discussion}

It has been shown that IncRNAs function primarily through their interactions with cellular macromolecules, such as chromatin DNA, proteins and RNAs [30-32]. In the present study, we elucidated that IncRNA CYTOR forms a trimeric complex with NCL and Sam68 through the specific motifs on EXON1 and activates the NF- $\mathrm{kB}$ signaling pathway to promote CRC EMT and progression (Fig. 8h).

Our clinical data also revealed that CYTOR was frequently up-regulated in CRC samples and that its overexpression was significantly associated with poor prognosis for CRC patients. A systematic analysis of online databases suggested that CYTOR could be considered a risk factor for recurrence and prognosis in CRC patients. 
A

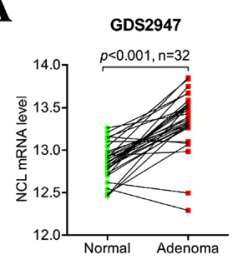

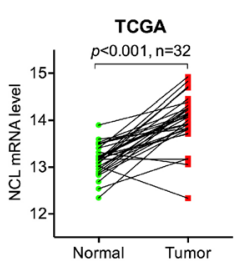
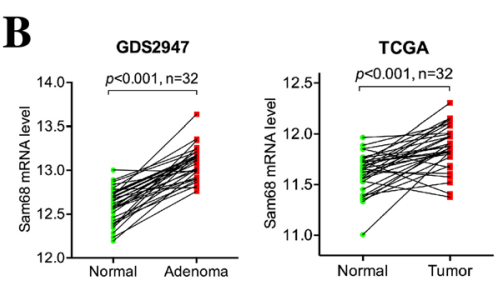

C
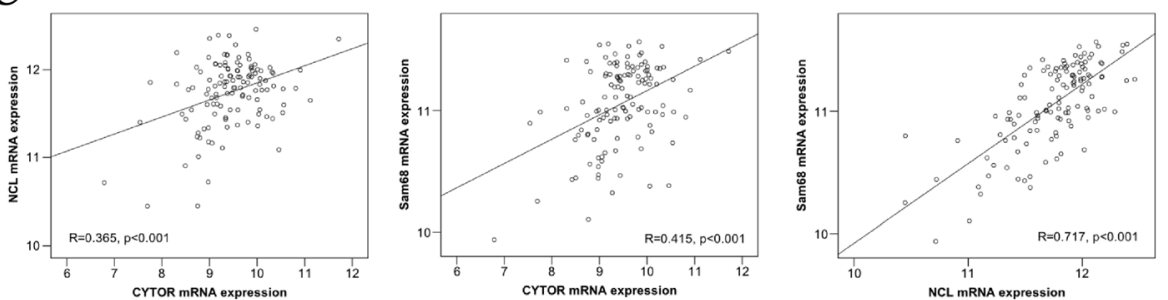

D

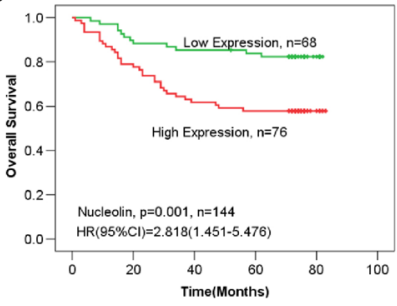

F
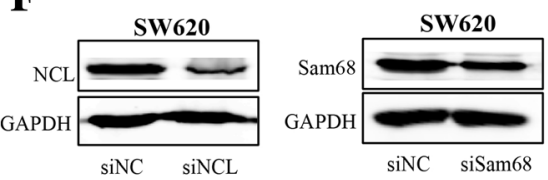

H
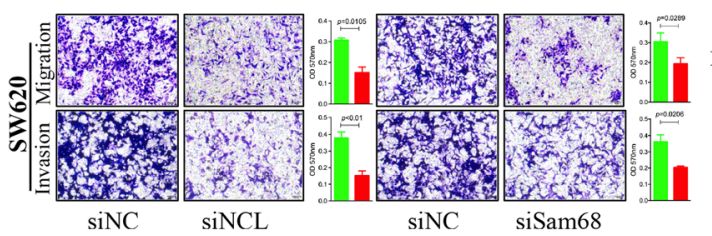

$\mathbf{E}$

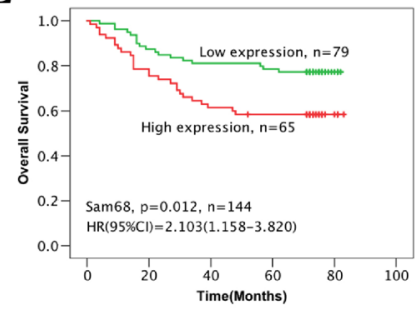

G

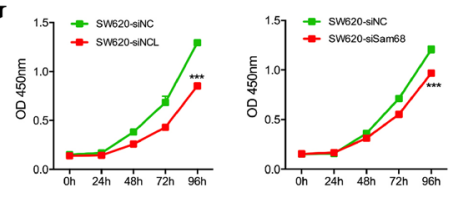

I

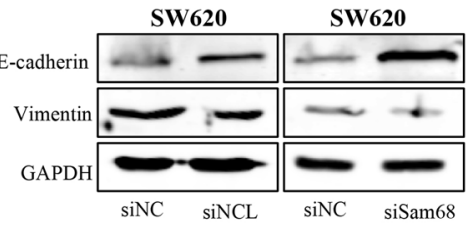

Fig. $7 \mathrm{NCL}$ and Sam68 acted as oncogenes and promoted CRC progression. a Higher expression of NCL in colorectal cancer than paired matched normal tissue samples from the GDS2947 and TCGA databases. b Higher expression of Sam68 in colorectal cancer than paired matched normal tissue samples from the GDS2947 and TCGA databases. c Positive correlation between CYTOR, NCL and Sam68 expression in the GSE38832 database. $\mathbf{d}$ Kaplan-Meier plots of overall survival versus NCL in CRC samples from our tissue bank, higher NCL expression with poorer survival. e Kaplan-Meier plots of overall survival versus Sam68 in CRC samples from our tissue bank, higher Sam68 expression with poorer survival. f Immunoblotting for detection of NCL and Sam68 in SW620 cells knocked down by siRNAs of NCL and Sam68. g Decrease of the proliferation ability for NCL knockdown (siNCL) and Sam68 knockdown (siSam68) SW620 cells compared with control (siNC) by CCK-8. $\mathbf{h}$ Decrease of migration/invasive potentials for NCL knockdown (siNCL) and Sam68 knockdown (siSam68) SW620 cells compared with control (siNC) by Transwell assay. i Expression of E-cadherin and Vimentin in NCL and Sam68 knockdown SW620 cells by immunoblotting

Our results indicated that CYTOR probably acts as a proto-oncogene in $\mathrm{CRC}$, which is similar to its role in lung cancer [33], gastric cancer [12], and other cancers. Mechanistically, CYTOR might function as a competing endogenous RNA to regulate tumorigenesis and progression. For example, CYTOR sponged miRNA-103a-3p to promote malignant progression of glioma stem cells [34], modulated the expression of miR-193a-3p to confer resistance to oxaliplatin in colon cancer [14], and negatively regulated miR-205 to promote renal cell carcinoma progression [35]. Proteins interacting with CYTOR had been investigated in other cancer types; for example, EZH2 and EGFR were reported to interact with CYTOR in gastric cancer [36, 37]. In our ChIRP and MS assays, EZH2 and EGFR were not detected; a possible explanation might be the different types of cancer cells used. 

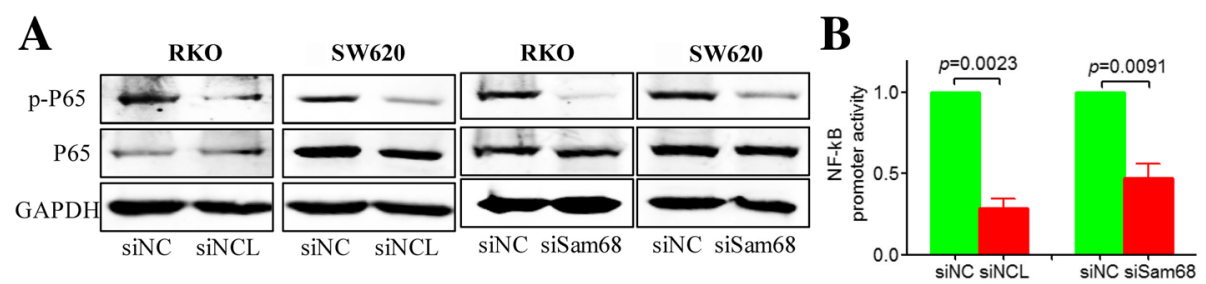

$\mathbf{C}$

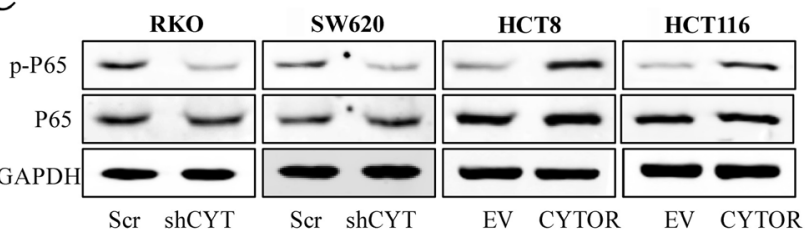

D

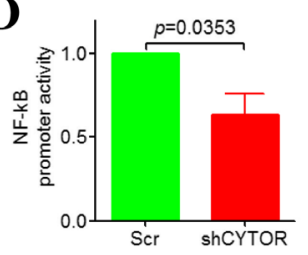

$\mathbf{E}$
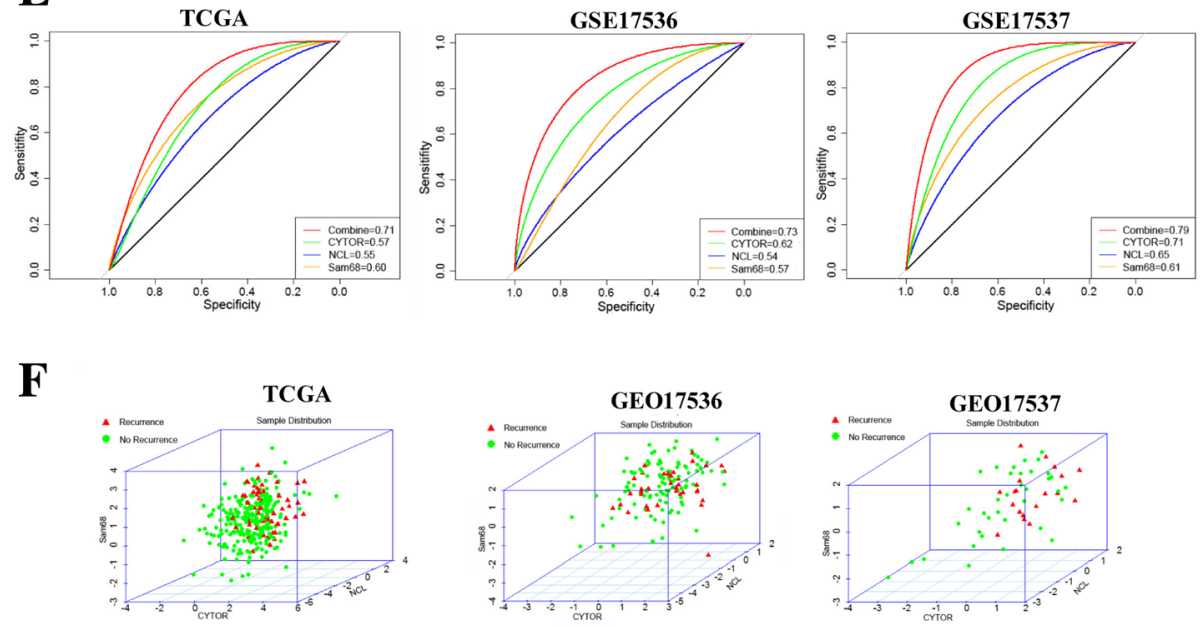

G
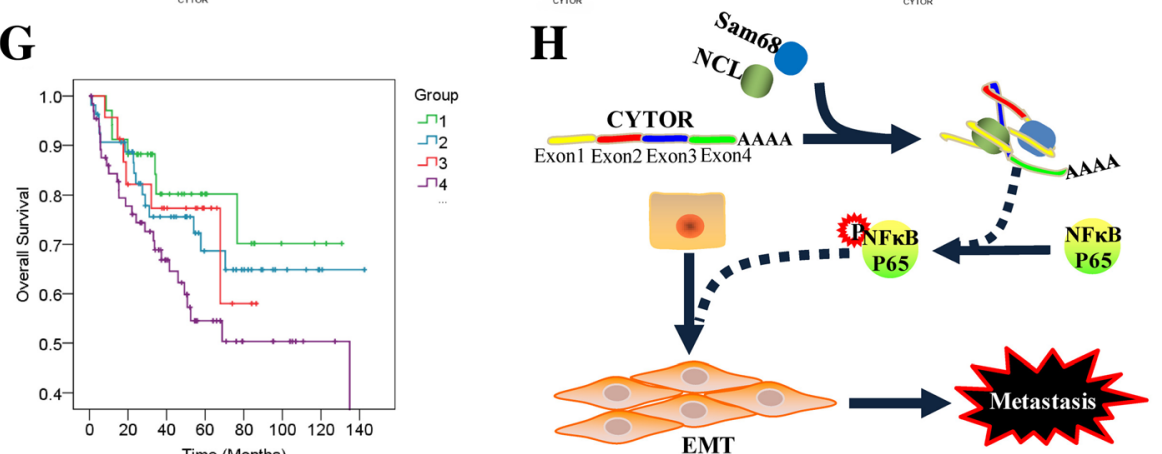

Fig. 8 The NCL-CYTOR-Sam68 complex activated the NF-KB pathway and acted as a prognostic biomarker. a Expression of P65 and phosphorylated P65 in NCL and Sam68 knockdown RKO and SW620 cells by immunoblotting. b Luciferase promoter reporter assays of NF-KB in NCL and Sam68 knockdown cells. c Expression of P65 and phosphorylated P65 in CYTOR knockdown cells and in CYTOR-overexpressing HCT8 and HCT116 cells by immunoblotting. $\mathbf{d}$ Luciferase promoter reporter assays of NF-KB in CYTOR knockdown cells. e ROC curve analysis for the combination of CYTOR, NCL and Sam68 in the TCGA, GSE17536 and GSE17537 databases. $\mathbf{f}$ 3D curve assay for the relationship between recurrence and the expression distribution of CYTOR, NCL and Sam68. g Kaplan-Meier plots of overall survival for the combination of CYTOR, NCL and Sam68 in GSE17536 database; group 1 has low expression for all three molecules; group 2 has low expression of CYTOR only; group 3 has high expression of CYTOR only; group 4 has high expression of all three molecules. $\mathbf{h}$ Schematic model of the function of CYTOR in CRC progression. CYTOR mediates the interaction of $\mathrm{NCL}$ and Sam68 through specific motifs in its EXON1 and activates the NF-KB signaling pathway to promote CRC EMT and metastasis

NCL, one of the two proteins identified, is an acidic phosphoprotein that is abundantly expressed in exponentially growing cells and is located mainly in the nucleolus; it can also be found in the nucleoplasm, cytoplasm, and cell membrane [17]. In addition to ribosome biogenesis, NCL contributes to cancer progression through shuttling 
between the nucleolus, cytoplasm and cell membrane and regulating BCL-2, P53 and MMP9, and other proteins [38]. Overexpression of NCL was found in several types of cancer, including lung and breast cancer [39]. In particular, ectopic expression of NCL in colorectal cancer was associated with higher aggressiveness and worse prognosis [39], consistent with our results. In this study, we showed that NCL could bind to a specific site in EXON1 of CYTOR, which might contribute to CRC progression and metastasis.

The other protein, SAM68, is a tyrosine-phosphorylated, SRC-associated protein that is present in mitotic cells and plays key roles during cell differentiation and development [18]. Aberrant expression of SAM68 was detected in several types of tumors such as prostate cancer, non-small cell lung cancer, renal cell carcinoma and colorectal cancer, in which high SAM68 expression was inversely associated with overall survival [18, 40]. Our current study also revealed that Sam68 could specifically recognize its binding site in EXON1 of CYTOR and, together with NCL, acted as an oncogene contributing to CRC progression. These data also suggested that EXON1 of CYTOR is the key functional motif that mediates the formation of the heterotrimeric complex.

Dysregulation of NF-KB signaling is a common event in many types of cancer and contributes to tumor initiation and progression by driving the expression of pro-proliferative/anti-apoptotic genes. More importantly, NF- $\mathrm{kB}$ signaling also plays critical roles in EMT and cancer progression [29]. In this study, we also demonstrated that the NCL-CYTOR-Sam68 complex could activate the NF- $\mathrm{kB}$ signaling pathway, thus promoting EMT and metastasis in CRC. Still, the underlying mechanism whereby CYTOR, NCL and Sam68 regulate the NF- $\mathrm{KB}$ signaling pathway needs further investigation.

\section{Conclusions}

We identified the functional roles played in CRC progression by CYTOR, which forms a heterotrimeric complex with NCL and Sam68 through EXON1. We also provided strong clinical evidence for CYTOR as a biomarker of recurrence and prognosis of CRC. In addition, on the basis of the important function of the NCL-CYTOR-Sam68 complex, these molecules might have potential as novel targets for CRC therapy in the future.

\section{Additional files}

Additional file 1: Supplementary Materials and Methods. (DOCX 33 kb)

Additional file 2: Figure S1. CYTOR expression and CRC prognosis.

(A, B) Kaplan-Meier plots of overall survival (A) and recurrence-free survival (B) for CRC samples from the GSE17536 database. (C, D) Kaplan-Meier plots of overall survival (C) and recurrence-free survival (D) for CRC samples from the GSE17537 database. (E, F) Kaplan-Meier plots of overall survival (E) and recurrence-free survival (F) for CRC samples from the GSE56699 database. $(G, H)$ Kaplan-Meier plots of overall survival for CRC samples from the GSE16125 (G) and GSE29621 (H) databases. (I, J, K, L) Kaplan-Meier plots of disease-free survival for CRC samples from the GSE24549-GPL11028 (I), GSE24549-GPL5175 (J), GSE24550-GPL11028 (K) and GSE24550-GPL5175 (L) databases. (M, N) Kaplan-Meier plots of recurrence-free survival for CRC samples from the GSE31595 (M) and GSE33113 (N) databases. (JPG 688 kb)

Additional file 3: Figure S2. A meta-analysis of the association between CYTOR and CRC survival. (A) Forest plots of the association between CYTOR expression and overall survival at the cutoff value set according to the ROC. (B) Forest plots of the association between CYTOR expression and disease- or recurrence-free survival at the cutoff value set according to the ROC. (C) Forest plots of the association between CYTOR expression and overall survival at the P50 cutoff value. (D) Forest plots of the association between CYTOR expression and disease- or recurrence-free survival at the P50 cutoff value. (JPG $1781 \mathrm{~kb}$ )

Additional file 4: Figure S3. Funnel plots for the relationship between CYTOR and CRC prognosis. (A) Funnel plots of the association between CYTOR expression and overall survival at the cutoff value set according to the ROC. (B) Funnel plots of the association between CYTOR expression and disease- or recurrence-free survival at the cutoff value set by according to the ROC. (C) Funnel plots of the association between CYTOR expression and overall survival at the P50 cutoff value. (D) Funnel plots of the association between CYTOR expression and disease- or recurrence-free survival at the P50 cutoff value. (JPG $1257 \mathrm{~kb}$ )

Additional file 5: Figure S4. Knockdown of CYTOR inhibited anchorage-independent growth and migration/invasion.(A) qRT-PCR for detection of CYTOR in RKO, SW480 and SW620 cells knocked known by siRNAs of CYTOR. (B) Reduction of colony formation ability for CYTOR knockdown RKO and SW620 cells by siRNAs compared with control (NC). (C, D and E) Decrease of migration/invasive potential for CYTOR knockdown RKO (C), SW480 (D) and SW620 (E) cells by siRNAs compared with control by transwell assay. (JPG $2970 \mathrm{~kb}$ )

Additional file 6: Figure S5. Correlation analysis between CYTOR and EMT markers. (JPG $567 \mathrm{~kb}$ )

Additional file 7: Figure S6. CYTOR location in cells and its binding proteins identified by ChIRP and MS. (A) RNA FISH to detection CYTOR location in RKO cells. (B) SDS-PAGE for protein isolation by ChIRP with CYTOR-specific probes. (C) MS identification of NCL and Sam68. (JPG $1204 \mathrm{~kb}$ )

Additional file 8: Figure S7. Expression and biological function of NCL and Sam68 in CRC. (A) Higher expression of NCL in colorectal cancer than paired matched normal tissue samples from the GSE31737, GSE32323 and GSE41328 databases. (B) Higher expression of Sam68 in colorectal cancer than paired matched normal tissue samples from the GSE32323 database. (C) Decrease of the proliferation ability for NCL knockdown (siNCL) and Sam68 knockdown (siSam68) RKO cells compared with control (siNC) by CCK8. (D) Decrease of migration/ invasive potentials for NCL knockdown (siNCL) and Sam68 knockdown (siSam68) RKO cells compared with control (siNC) by Transwell assay. (JPG $2659 \mathrm{~kb}$ )

Additional file 9: Figure S8. Correlation analysis of NCL, Sam68 and EMT markers in GEO GSE38832 database. (A) Correlation between NCL and EMT markers. (B) Correlation between Sam68 and EMT markers. (JPG $432 \mathrm{~kb})$

\section{Abbreviations}

CCAL: colorectal cancer-associated IncRNA; CCAT1: colon cancer associated transcript 1; ChIRP-MS: Chromatin Isolation by RNA Purification-mass spectrometry; co-IP: Co-Immunoprecipitation; CRC: colorectal cancer; CYTOR: cytoskeleton regulator RNA/Linc00152; DFS: disease-free survival; EGFR: epidermal growth factor receptor; EMT: Epithelial-mesenchymal transition; EZH2: enhancer of zeste 2 polycomb repressive complex 2 subunit; FISH: RNA fluorescence in situ hybridization; FL: Full length; FN1: fibronectin 1; GEO: gene expression omnibus; HOTAIR: HOX transcript antisense RNA; IHC: immunohistochemistry; IncRNAs: Long non-coding RNAs; MMP9: matrix metallopeptidase 9; NCL: Nucleolin; OS: overall survival; qRT-PCR: Quantitative-Reverse transcription 
polymerase chain reaction-Reverse transcription polymerase chain reaction; RBDs: RNA binding domains; RBPs: RNA-binding proteins; RFS: recurrence-free survival; RIP: RNA immunoprecipitation; RT-PCR: Reverse transcription polymerase chainreaction; Sam68: KH RNA binding domain containing, signal transduction associated 1/KHDRBS1; TCGA: The Cancer Genome Atlas; WT: wild-type; $\triangle \mathrm{C}$ : C-terminus-deleted; $\triangle$ EXON1: EXON1 deletion; $\triangle$ EXON4: EXON4 deletion; $\triangle \mathrm{KH}$ : $\mathrm{KH}$-domain-deleted; $\Delta \mathrm{N}$ : N-terminus-deleted

\section{Acknowledgements}

We thank Ms. Qiong Huang (Zhejiang University) for technical support of this study.

\section{Funding}

This work is supported by grants from the National Natural Science Foundation of China (81672730, 81602073 and 81572716), the 111 project (B13026), the Natural Science Foundation of Zhejiang Province (LY17H160025) and Department of Science and Technology of Zhejiang Province (2016C33150).

\section{Availability of data and materials}

The data supporting the conclusions of this article are included within main text and the supplementary materials.

\section{Authors' contributions}

$\mathrm{HZ}$ and ML conceived, designed and supervised the study; $\mathrm{HZ}$ and XW interpreted the results; $\mathrm{HZ}, \mathrm{XW}$ and $\mathrm{HY}$ drafted the manuscript; XW, HY, WS, $\mathrm{JK}$ and LZ performed all experiments; JT and JW interpreted the results of $I H C$, WS and EX performed statistical analyses. All authors read and approved the final manuscript.

\section{Ethics approval and consent to participate}

The protocols were approved by the Internal Review Board of Zhejiang University (2016007)

\section{Consent for publication}

All authors give consent for the publication of the manuscript in Molecular Cancer.

\section{Competing interests}

The authors declare no conflict of interest.

\section{Publisher's Note}

Springer Nature remains neutral with regard to jurisdictional claims in published maps and institutional affiliations.

\section{Author details}

${ }^{1}$ Department of Pathology, Key Laboratory of Disease Proteomics of Zhejiang Province, Zhejiang University School of Medicine, Hangzhou 310058, China. 2Department of Pharmacology, China Pharmaceutical University, Nanjing 210009, China.

Received: 24 November 2017 Accepted: 20 July 2018

Published online: 31 July 2018

\section{References}

1. Hanahan D, Weinberg RA. Hallmarks of cancer: the next generation. Cell. 2011;144:646-74

2. Tomasetti C, Li L, Vogelstein B. Stem cell divisions, somatic mutations, cancer etiology, and cancer prevention. Science. 2017;355:1330-4.

3. Bhan A, Soleimani M, Mandal SS. Long noncoding RNA and Cancer: a new paradigm. Cancer Res. 2017;77:3965-81.

4. Reimers MS, Zeestraten EC, Kuppen PJ, Liefers GJ, van de Velde CJ. Biomarkers in precision therapy in colorectal cancer. Gastroenterol Rep (Oxf). 2013;1:166-83.

5. Taniue K, Kurimoto A, Sugimasa H, Nasu E, Takeda Y, Iwasaki K, Nagashima T, Okada-Hatakeyama M, Oyama M, Kozuka-Hata H, et al. Long noncoding RNA UPAT promotes colon tumorigenesis by inhibiting degradation of UHRF1. Proc Natl Acad Sci U S A. 2016;113:1273-8.

6. McCleland ML, Mesh K, Lorenzana E, Chopra VS, Segal E, Watanabe C, Haley B, Mayba O, Yaylaoglu M, Gnad F, Firestein R. CCAT1 is an enhancertemplated RNA that predicts BET sensitivity in colorectal cancer. J Clin Invest. 2016;126:639-52.
7. Ma Y, Yang Y, Wang F, Moyer MP, Wei Q, Zhang P, Yang Z, Liu W, Zhang H, Chen $\mathrm{N}$, et al. Long non-coding RNA CCAL regulates colorectal cancer progression by activating Wnt/beta-catenin signalling pathway via suppression of activator protein 2alpha. Gut. 2016;65:1494-504.

8. Kong J, Sun W, Li C, Wan L, Wang S, Wu Y, Xu E, Zhang H, Lai M. Long non-coding RNA LINC01133 inhibits epithelial-mesenchymal transition and metastasis in colorectal cancer by interacting with SRSF6. Cancer Lett. 2016; 380:476-84.

9. Yang F, Huo XS, Yuan SX, Zhang L, Zhou WP, Wang F, Sun SH. Repression of the long noncoding RNA-LET by histone deacetylase 3 contributes to hypoxia-mediated metastasis. Mol Cell. 2013;49:1083-96.

10. Kogo R, Shimamura T, Mimori K, Kawahara K, Imoto S, Sudo T, Tanaka F, Shibata K, Suzuki A, Komune S, et al. Long noncoding RNA HOTAIR regulates polycomb-dependent chromatin modification and is associated with poor prognosis in colorectal cancers. Cancer Res. 2011;71:6320-6.

11. Li H, Zhong A, Li S, Meng X, Wang X, Xu F, Lai M. The integrated pathway of TGFbeta/snail with TNFalpha/NFkappaB may facilitate the tumor-stroma interaction in the EMT process and colorectal cancer prognosis. Sci Rep. 2017;7:4915.

12. Zhao J, Liu Y, Zhang W, Zhou Z, Wu J, Cui P, Zhang Y, Huang G. Long non-coding RNA Linc00152 is involved in cell cycle arrest, apoptosis, epithelial to mesenchymal transition, cell migration and invasion in gastric cancer. Cell Cycle. 2015;14:3112-23.

13. Li J, Wang X, Tang J, Jiang R, Zhang W, Ji J, Sun B. HULC and Linc00152 act as novel biomarkers in predicting diagnosis of hepatocellular carcinoma. Cell Physiol Biochem. 2015;37:687-96.

14. Yue B, Cai D, Liu C, Fang C, Yan D. Linc00152 functions as a competing endogenous RNA to confer Oxaliplatin resistance and holds prognostic values in Colon Cancer. Mol Ther. 2016;24:2064-77.

15. Cai Q, Wang ZQ, Wang SH, Li C, Zhu ZG, Quan ZW, Zhang WJ. Upregulation of long non-coding RNA LINC00152 by SP1 contributes to gallbladder cancer cell growth and tumor metastasis via PI3K/AKT pathway. Am J Trans Res. 2016;8:4068-81

16. Wu Y, Tan C, Weng WW, Deng Y, Zhang QY, Yang XQ, Gan HL, Wang T, Zhang PP, Xu MD, et al. Long non-coding RNA Linc00152 is a positive prognostic factor for and demonstrates malignant biological behavior in clear cell renal cell carcinoma. Am J Cancer Res. 2016;6:285-99.

17. Jia W, Yao Z, Zhao J, Guan Q, Gao L. New perspectives of physiological and pathological functions of nucleolin (NCL). Life Sci. 2017;186:1-10.

18. Bielli P, Busa R, Paronetto MP, Sette C. The RNA-binding protein Sam68 is a multifunctional player in human cancer. Endocr Relat Cancer. 2011;18:R91-R102.

19. Jensen DH, Dabelsteen E, Specht L, Fiehn AM, Therkildsen MH, Jonson L, Vikesaa J, Nielsen FC, von Buchwald C. Molecular profiling of tumour budding implicates TGFbeta-mediated epithelial-mesenchymal transition as a therapeutic target in oral squamous cell carcinoma. J Pathol. 2015:236:505-16.

20. De Smedt L, Palmans S, Andel D, Govaere O, Boeckx B, Smeets D, Galle E, Wouters J, Barras D, Suffiotti M, et al. Expression profiling of budding cells in colorectal cancer reveals an EMT-like phenotype and molecular subtype switching. Br J Cancer. 2017:116:58-65.

21. Tan X, Banerjee P, Guo HF, Ireland S, Pankova D, Ahn YH, Nikolaidis IM, Liu X, Zhao Y, Xue Y, et al. Epithelial-to-mesenchymal transition drives a pro-metastatic Golgi compaction process through scaffolding protein PAQR11. J Clin Invest. 2017:127:117-31.

22. Chu C, Quinn J, Chang HY. Chromatin isolation by RNA purification (ChIRP). J Vis Exp. 2012:61:e3912-7.

23. Farin K, Di Segni A, Mor A, Pinkas-Kramarski R. Structure-function analysis of nucleolin and ErbB receptors interactions. PLoS One. 2009;4:e6128.

24. Fu K, Sun X, Wier EM, Hodgson A, Liu Y, Sears CL, Wan F. Sam68/KHDRBS1 is critical for colon tumorigenesis by regulating genotoxic stress-induced NF-kappaB activation. Elife. 2016;5:e15018-59.

25. Mathews DH, Disney MD, Childs JL, Schroeder SJ, Zuker M, Turner DH. Incorporating chemical modification constraints into a dynamic programming algorithm for prediction of RNA secondary structure. Proc Natl Acad Sci U S A. 2004:101:7287-92

26. Finger LD, Trantirek $L$, Johansson C, Feigon J. Solution structures of stemloop RNAs that bind to the two N-terminal RNA-binding domains of nucleolin. Nucleic Acids Res. 2003;31:6461-72.

27. Feracci M, Foot JN, Grellscheid SN, Danilenko M, Stehle R, Gonchar O, Kang HS, Dalgliesh C, Meyer NH, Liu Y, et al. Structural basis of RNA recognition and dimerization by the STAR proteins T-STAR and Sam68. Nat Commun. 2016;7:10355 
28. Vaiopoulos AG, Athanasoula K, Papavassiliou AG. NF-kappaB in colorectal cancer. J Mol Med (Berl). 2013;91:1029-37.

29. Min C, Eddy SF, Sherr DH, Sonenshein GE. NF-kappaB and epithelial to mesenchymal transition of cancer. J Cell Biochem. 2008:104:733-44.

30. Wang KC, Chang HY. Molecular mechanisms of long noncoding RNAs. Mol Cell. 2011:43:904-14.

31. Bohmdorfer G, Wierzbicki AT. Control of chromatin structure by long noncoding RNA. Trends Cell Biol. 2015;25:623-32.

32. Bonasio R, Shiekhattar R. Regulation of transcription by long noncoding RNAs. Annu Rev Genet. 2014;48:433-55.

33. Chen QN, Chen X, Chen ZY, Nie FQ, Wei CC, Ma HW, Wan L, Yan S, Ren SN, Wang ZX. Long intergenic non-coding RNA 00152 promotes lung adenocarcinoma proliferation via interacting with EZH2 and repressing IL24 expression. Mol Cancer. 2017;16:17.

34. Yu M, Xue Y, Zheng J, Liu X, Yu H, Liu L, Li Z, Liu Y. Linc00152 promotes malignant progression of glioma stem cells by regulating miR-103a-3p/FEZF1/CDC25A pathway. Mol Cancer. 2017;16:110.

35. Wang Y, Liu J, Bai H, Dang Y, Lv P, Wu S. Long intergenic non-coding RNA 00152 promotes renal cell carcinoma progression by epigenetically suppressing P16 and negatively regulates miR-205. Am J Cancer Res. 2017;7:312-22.

36. Chen WM, Huang MD, Sun DP, Kong R, Xu TP, Xia R, Zhang EB, Shu YQ. Long intergenic non-coding RNA 00152 promotes tumor cell cycle progression by binding to EZH2 and repressing p15 and p21 in gastric cancer. Oncotarget. 2016;7:9773-87.

37. Zhou J, Zhi X, Wang L, Wang W, Li Z, Tang J, Wang J, Zhang Q, Xu Z. Linc00152 promotes proliferation in gastric cancer through the EGFRdependent pathway. J Exp Clin Cancer Res. 2015;34:135.

38. Abdelmohsen K, Gorospe M. RNA-binding protein nucleolin in disease. RNA Biol. 2012;9:799-808.

39. Berger CM, Gaume X, Bouvet P. The roles of nucleolin subcellular localization in cancer. Biochimie. 2015;113:78-85.

40. Frisone P, Pradella D, Di Matteo A, Belloni E, Ghigna C, Paronetto MP. SAM68: signal transduction and RNA metabolism in human Cancer. Biomed Res Int. 2015;2015:528954.

Ready to submit your research? Choose BMC and benefit from:

- fast, convenient online submission

- thorough peer review by experienced researchers in your field

- rapid publication on acceptance

- support for research data, including large and complex data types

- gold Open Access which fosters wider collaboration and increased citations

- maximum visibility for your research: over $100 \mathrm{M}$ website views per year

At $\mathrm{BMC}$, research is always in progress.

Learn more biomedcentral.com/submissions 